\title{
Correction: LSD1 inhibition by tranylcypromine derivatives interferes with GFI1-mediated repression of PU.1 target genes and induces differentiation in AML
}

\author{
Jessica Barth ${ }^{1,2,3} \cdot$ Khalil Abou-El-Ardat $^{1,2,3} \cdot$ Denis Dalic $^{1} \cdot$ Nina Kurrle $^{1} \cdot$ Anna-Maria Maier $^{1} \cdot$ Sebastian Mohr ${ }^{1}$. \\ Judith Schütte $^{4} \cdot$ Lothar Vassen $^{4} \cdot$ Gabriele Greve $^{3,5,6}$ - Johannes Schulz-Fincke ${ }^{3,5,7}$ - Martin Schmitt ${ }^{7}$ Milica Tosic $^{8}$. \\ Eric Metzger $^{8} \cdot$ Gesine Bug $^{1,2,3} \cdot$ Cyrus Khandanpour $\mathbb{1}^{4} \cdot$ Sebastian A. Wagner ${ }^{1,2,3} \cdot$ Michael Lübbert $^{3,5,6}$. \\ Manfred Jung ${ }^{3,5,7} \cdot$ Hubert Serve $^{1,2,3} \cdot$ Roland Schüle ${ }^{8,9} \cdot$ Tobias Berg $^{1,2,3}$
}

Published online: 5 March 2019

(c) Springer Nature Limited 2019

\section{Correction to: Leukemia;}

https://doi.org/10.1038/s41375-018-0375-7;

published online 24 January 2019

The original version of this Article contained an error in the Acknowledgements section.
The final sentence "ML, RS and MJ were supported by the DFG CRC 992 (MEDEP)" contained a typo in the initials of author RS, and was originally written as "ML, JS and MJ were supported by the DFG CRC 992 (MEDEP)."

This has now been corrected in both the PDF and HTML versions of the Article.

Tobias Berg

tobias.berg@kgu.de

1 Department of Medicine II, Hematology/Oncology, Goethe University, Frankfurt/Main, Germany

2 German Cancer Consortium (DKTK), Frankfurt, Germany

3 German Cancer Research Center (DKFZ), Heidelberg, Germany

4 Department of Medicine A, University Hospital Muenster, Muenster, Germany

5 German Cancer Consortium (DKTK), Freiburg, Germany

6 Department of Medicine I, Hematology, Oncology and Stem Cell Transplantation, Faculty of Medicine, University of Freiburg Medical Center, Freiburg, Germany

7 Institute of Pharmaceutical Sciences, University of Freiburg, Freiburg, Germany

8 Department of Urology and Center for Clinical Research, University of Freiburg Medical Center, Freiburg, Germany

9 BIOSS Centre for Biological Signalling Studies, Albert-LudwigsUniversity, 79104 Freiburg, Germany 